\title{
UK Renal Registry 18th Annual Report: Appendix H Coding: Ethnicity, EDTA Primary Renal Diagnoses, EDTA Causes of Death
}

\section{H1: Ethnicity coding}

In some renal centres ethnicity data is recorded in the clinical information systems in the individual renal centres in the format of 9S. . read codes. In other centres it is extracted from local PAS systems in a different format and should be recoded to the 9S. . format by the centre, before being sent to the UK Renal Registry (UKRR). For report analyses, ethnic categories are condensed into five groups (White, South Asian, Black, Chinese and Other). For some analyses Chinese are grouped into Other.

\begin{tabular}{|c|c|c|c|c|}
\hline Read code & Ethnic category & Assigned group & Old PAS & New PAS \\
\hline 9S1.. & White & White & 0 & A1 \\
\hline 9SA9. & Irish (NMO) & White & & B1 \\
\hline 9SAA. & Greek Cypriot (NMO) & White & & CG \\
\hline 9SAB. & Turkish Cypriot (NMO) & White & & CJ \\
\hline 9SAC. & Other European (NMO) & White & & $\mathrm{C} 1$ \\
\hline 9S6.. & Indian & S Asian & 4 & $\mathrm{H} 1$ \\
\hline 9S7.. & Pakistani & S Asian & 5 & $\mathrm{~J} 1$ \\
\hline 9S8. & Bangladeshi & S Asian & 6 & K1 \\
\hline 9SA6. & East African Asian & S Asian & & \\
\hline 9SA7. & Indian Subcontinent & S Asian & & \\
\hline 9SA8. & Other Asian & S Asian & & L1 \\
\hline 9S2.. & Black Caribbean & Black & 1 & M1 \\
\hline 9S3.. & Black African & Black & 2 & N1 \\
\hline 9S4.. & Black/Other/non-mixed origin & Black & 3 & P1 \\
\hline 9S41. & Black British & Black & & $\mathrm{PD}$ \\
\hline $9 S 42$. & Black Caribbean & Black & & \\
\hline $9 S 43$. & Black North African & Black & & \\
\hline $9 S 44$. & Black other African country & Black & & \\
\hline $9 S 45$. & Black East African Asian & Black & & \\
\hline $9 S 46$. & Black Indian subcontinent & Black & & \\
\hline 9 S47. & Black Other Asian & Black & & \\
\hline 9S48. & Black Black Other & Black & & $\mathrm{PE}$ \\
\hline 9S5.. & Black other/mixed & Black & & \\
\hline 9S51. & Other Black Black/White origin & Black & & GC \\
\hline 9S52. & Other Black - Black/Asian origin & Black & & GA \\
\hline 9S9.. & Chinese & Chinese & 7 & R1 \\
\hline 9T1C. & Chinese & Chinese & & \\
\hline 9SA.. & Other ethnic non-mixed (NMO) & Other & & \\
\hline 9SA1. & British ethnic minority specified (NMO) & Other & & \\
\hline
\end{tabular}

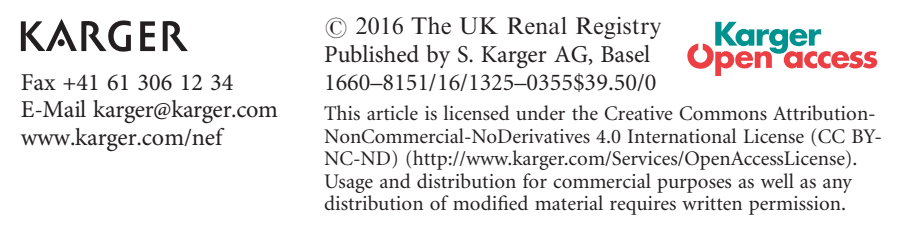




\begin{tabular}{|c|c|c|c|c|}
\hline Read code & Ethnic category & Assigned group & Old PAS & New PAS \\
\hline 9SA2. & British ethnic minority unspecified (NMO) & Other & & \\
\hline 9SA3. & Caribbean Island (NMO) & Other & & \\
\hline 9SA5. & Other African countries (NMO) & Other & & \\
\hline 9SAD. & Other ethnic NEC (NMO) & Other & & S1 \\
\hline 9SB.. & Other ethnic/mixed origin & Other & 8 & \\
\hline 9SB3. & Other ethnic/mixed White origin & Other & & \\
\hline 9SB4. & Other ethnic/Other mixed origin & Other & & G1 \\
\hline
\end{tabular}

NMO denotes non-mixed origin

\section{H2: EDTA primary renal diagnoses}

New primary renal diagnosis codes (PRD) were produced in 2012 [1]. The data used for this report included a mixture of old and new ERA-EDTA codes. The split was about 50:50 for 2014 incident patients. For those people without an old code, new codes (where available) were mapped back to old codes using the mapping available on the ERA-EDTA website. As recommended in the notes for users in the ERA-EDTA's PRD code list document the mapping of new to old codes is provided for guidance only and has not been validated; therefore care must be taken not to over interpret data from this mapping.

The old codes (both those received from centres and those mapped back from new codes) were then grouped into the same eight categories as in previous reports as shown in the table below.

\begin{tabular}{|c|c|c|}
\hline EDTA code & Title & UKRR category \\
\hline 0 & Chronic renal failure; aetiology uncertain unknown/unavailable & Uncertain \\
\hline 10 & Glomerulonephritis; histologically NOT examined & Glomerulonephritis* \\
\hline 11 & Focal segmental glomerulosclerosis with nephrotic syndrome in children & Glomerulonephritis \\
\hline 12 & IgA nephropathy (proven by immunofluorescence, not code 76 and not 85 ) & Glomerulonephritis \\
\hline 13 & $\begin{array}{l}\text { Dense deposit disease; membrano-proliferative GN; type II (proven by immunofluorescence } \\
\text { and/or electron microscopy) }\end{array}$ & Glomerulonephritis \\
\hline 14 & Membranous nephropathy & Glomerulonephritis \\
\hline 15 & $\begin{array}{l}\text { Membrano-proliferative GN; type I (proven by immunofluorescence and/or electron } \\
\text { microscopy - not code } 84 \text { or } 89 \text { ) }\end{array}$ & Glomerulonephritis \\
\hline 16 & Crescentic (extracapillary) glomerulonephritis (type I, II, III) & Glomerulonephritis \\
\hline 17 & Focal segmental glomerulosclerosis with nephrotic syndrome in adults & Glomerulonephritis \\
\hline 19 & Glomerulonephritis; histologically examined, not given above & Glomerulonephritis \\
\hline 20 & Pyelonephritis - cause not specified & Pyelonephritis \\
\hline 21 & Pyelonephritis associated with neurogenic bladder & Pyelonephritis \\
\hline 22 & Pyelonephritis due to congenital obstructive uropathy with/without vesico-ureteric reflux & Pyelonephritis \\
\hline 23 & Pyelonephritis due to acquired obstructive uropathy & Pyelonephritis \\
\hline 24 & Pyelonephritis due to vesico-ureteric reflux without obstruction & Pyelonephritis \\
\hline 25 & Pyelonephritis due to urolithiasis & Pyelonephritis \\
\hline 29 & Pyelonephritis due to other cause & Pyelonephritis \\
\hline 30 & $\begin{array}{l}\text { Interstitial nephritis (not pyelonephritis) due to other cause, or unspecified (not mentioned } \\
\text { above) }\end{array}$ & Other \\
\hline 31 & Nephropathy (interstitial) due to analgesic drugs & Other \\
\hline 32 & Nephropathy (interstitial) due to cis-platinum & Other \\
\hline 33 & Nephropathy (interstitial) due to cyclosporin A & Other \\
\hline 34 & Lead induced nephropathy (interstitial) & Other \\
\hline 39 & Drug induced nephropathy (interstitial) not mentioned above & Other \\
\hline 40 & Cystic kidney disease - type unspecified & Polycystic \\
\hline 41 & Polycystic kidneys; adult type (dominant) & Polycystic \\
\hline 42 & Polycystic kidneys; infantile (recessive) & Polycystic \\
\hline 43 & Medullary cystic disease; including nephronophtisis & Other \\
\hline
\end{tabular}


Cystic kidney disease - other specified type

Other

Hereditary/Familial nephropathy - type unspecified

Other

Hereditary nephritis with nerve deafness (Alport's Syndrome)

Other

Cystinosis

Primary oxalosis

Other

Fabry's disease

Other

Hereditary nephropathy - other specified type

Other

Renal hypoplasia (congenital) - type unspecified

Other

Oligomeganephronic hypoplasia

Congenital renal dysplasia with or without urinary tract malformation

Other

Other

Syndrome of agenesis of abdominal muscles (Prune Belly)

Renal vascular disease - type unspecified

Renal vascular disease due to malignant hypertension

Renal vascular disease due to hypertension

Renal vascular disease due to polyarteritis

Wegener's granulomatosis

Ischaemic renal disease/cholesterol embolism

Glomerulonephritis related to liver cirrhosis

Cryoglobulinemic glomerulonephritis

Renal vascular disease - due to other cause (not given above and not code 84-88)

Type 1 diabetes with diabetic nephropathy

Type 2 diabetes with diabetic nephropathy

Myelomatosis/light chain deposit disease

Other

Other

Renal vascular disease

Hypertension

Hypertension

Renal vascular disease

Other

Renal vascular disease

Other

Other

Renal vascular disease

Diabetes

Diabetes

Other

Other

Other

Lupus erythematosus

Henoch-Schoenlein purpura

Other

Goodpasture's syndrome

Systemic sclerosis (scleroderma)

Haemolytic Ureaemic Syndrome (including Moschcowitz syndrome)

Other

Other

Other

Multi-system disease - other (not mentioned above)

Other

Tubular necrosis (irreversible) or cortical necrosis (different from 88)

Other

Tuberculosis

Other

Gout nephropathy (urate)

Other

Nephrocalcinosis and hypercalcaemic nephropathy

Other

Balkan nephropathy

Other

Kidney tumour

Other

Traumatic or surgical loss of kidney

Other

Not known

Other identified renal disorders

Missing

Other

Code not sent

Missing

*Prior to the 15th Annual Report categorised as 'uncertain'

\section{H3: EDTA cause of death}

\begin{tabular}{lll}
\hline EDTA code & Cause & UKRR category \\
\hline 0 & Cause of death uncertain/not determined & Uncert \\
11 & Myocardial ischaemia and infarction & Heart \\
12 & Hyperkalaemia & Other \\
13 & Haemorrhagic pericarditis & Other \\
14 & Other causes of cardiac failure & Heart \\
15 & Cardiac arrest/sudden death; other cause or unknown & Heart \\
16 & Hypertensive cardiac failure & Heart \\
17 & Hypokalaemia & Other \\
18 & Fluid overload/pulmonary oedema & Heart \\
21 & Pulmonary embolus & Other
\end{tabular}




\begin{tabular}{|c|c|c|}
\hline EDTA code & Cause & UKRR category \\
\hline 22 & Cerebro-vascular accident, other cause or unspecified & CVA \\
\hline 23 & Gastro-intestinal haemorrhage (digestive) & Other \\
\hline 24 & Haemorrhage from graft site & Other \\
\hline 25 & Haemorrhage from vascular access or dialysis circuit & Other \\
\hline 26 & Haemorrhage from ruptured vascular aneurysm (not code 22 or 23 ) & Other \\
\hline 27 & Haemorrhage from surgery (not codes $23,24,26$ ) & Other \\
\hline 28 & Other haemorrhage, (not codes 2327) & Other \\
\hline 29 & Mesenteric infarction & Other \\
\hline 31 & Pulmonary infection bacterial (not code 73 ) & Infect \\
\hline 32 & Pulmonary infection (viral) & Infect \\
\hline 33 & Pulmonary infection (fungal or protozoal; parasitic) & Infect \\
\hline 34 & Infections elsewhere except viral hepatitis & Infect \\
\hline 35 & Septicaemia & Infect \\
\hline 36 & Tuberculosis (lung) & Infect \\
\hline 37 & Tuberculosis (elsewhere) & Infect \\
\hline 38 & Generalized viral infection & Infect \\
\hline 39 & Peritonitis (all causes except for peritoneal dialysis) & Infect \\
\hline 41 & Liver disease due to hepatitis $B$ virus & Other \\
\hline 42 & Liver disease due to other viral hepatitis & Other \\
\hline 43 & Liver disease due to drug toxicity & Other \\
\hline 44 & Cirrhosis not viral (alcoholic or other cause) & Other \\
\hline 45 & Cystic liver disease & Other \\
\hline 46 & Liver failure cause unknown & Other \\
\hline 47 & Patient refused further treatment for end stage renal failure (ESRF) & Trt_stop \\
\hline 51 & Patient refused further treatment for end stage renal failure (ESRF) & Trt_stop \\
\hline 52 & Suicide & Other \\
\hline 53 & ESRF treatment ceased for any other reason & Trt_stop \\
\hline 54 & ESRF treatment withdrawn for medical reasons & Trt_stop \\
\hline 61 & Uraemia caused by graft failure & Trt_stop \\
\hline 62 & Pancreatitis & Other \\
\hline 63 & Bone marrow depression (Aplasia) & Other \\
\hline 64 & Cachexia & Other \\
\hline 66 & Malignant disease in patient treated by immunosuppressive therapy & Malignant \\
\hline 67 & Malignant disease: solid tumours except those of 66 & Malignant \\
\hline 68 & Malignant disease: lymphoproliferative disorders (Except 66) & Malignant \\
\hline 69 & Dementia & Other \\
\hline 70 & Peritonitis (sclerosing, with peritoneal dialysis) & Other \\
\hline 71 & Perforation of peptic ulcer & Other \\
\hline 72 & Perforation of colon & Other \\
\hline 73 & Chronic obstructive pulmonary disease & Other \\
\hline 81 & Accident related to ESRF treatment (not 25) & Other \\
\hline 82 & Accident unrelated to ESRF treatment & Other \\
\hline 90 & Uraemia caused by graft failure & Trt_stop \\
\hline 99 & Other identified cause of death & Othere* \\
\hline 100 & Peritonitis (bacterial, with peritoneal dialysis) & Infect \\
\hline 101 & Peritonitis (fungal, with peritoneal dialysis) & Infect \\
\hline 102 & Peritonitis (due to other cause, with peritoneal dialysis) & Infect \\
\hline
\end{tabular}

*Prior to the 15th Annual Report categorised as 'uncertain'

\section{Reference}

1 Venkat-Raman G et al. New Primary diagnosis codes for the ERA-EDTA.

Nephrol Dial Transplant 2012;27(12):4414-9 